\title{
COMPOSIÇÃO QUÍMICA E ATIVIDADE ANTINOCICEPTIVA EM MODELO ANIMAL DO ÓLEO ESSENCIAL DE Myrcia rostrata DC. (MYRTACEAE)
}

\author{
Aline do Nascimento Silva ${ }^{\mathrm{a}, *}$, Horácio Freitas Bomfim ${ }^{\mathrm{b}}$, Acsa Oliveira Magalhães ${ }^{\mathrm{a}}$, Marilene Lopes da Rocha ${ }^{\mathrm{a}}$ e Angélica \\ Maria Lucchese ${ }^{b}$

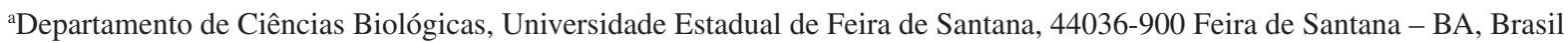 \\ b Departamento de Ciências Exatas, Universidade Estadual de Feira de Santana, 44036-900 Feira de Santana - BA, Brasil
}

Recebido em 18/03/2018; aceito em 30/07/2018; publicado na web em 21/08/2018

\begin{abstract}
CHEMICAL COMPOSITION AND ANTINOCICEPTIVE ACTIVITY OF ESSENTIAL OIL FROM Myrcia rostrata DC. (MYRTACEAE) IN ANIMAL MODELS. There are approximately 300 Myrcia DC. species in Brazil. The antimicrobial, antiinflammatory and antinociceptive activities of some of these species have been described in the literature. The present study aimed to analyze the chemical composition and evaluate the antinociceptive activity of essential oil from fresh Myrcia rostrata DC. leaves. The oil was obtained by hydrodistillation in a Clevenger-type apparatus and chemical analysis was conducted by GC/FID and GC/ MS. The acute oral toxicity and the rota rod tests were executed beforehand. The nociceptive tests (acetic acid-induced writhing, formalin and hot plate) were performed with the oil at doses of 75, 150 or $300 \mathrm{mg} \mathrm{kg}^{-1}$. Of the constituents identified, $95.24 \%$ were sesquiterpenes, carotol (17.68\%) being the major compound. The essential oil caused no obvious signs of toxicity, nor did it affect the motor coordination of the animals. In conclusion, the animals that received the oil, especially at $300 \mathrm{mg} \mathrm{kg}^{-1}$, demonstrated a lower number of writhes (1.62 versus 27.88 \pm 2.75 ), a decrease in paw-licking time in both phases of the formalin test, and increased latency on the hot plate when compared to the control group.
\end{abstract}

Keywords: sesquiterpenes; pain; inflammation.

\section{INTRODUÇÃO}

A dor é uma experiência sensorial e emocional, essas características, a fazem difícil de ser definida com exatidão. ${ }^{1}$ É um processo fisiológico e protetor, uma vez que atua como um sistema de alerta associado não somente a lesões reais como também potenciais. ${ }^{2}$

Mesmo sendo um mecanismo de defesa natural, a persistência dos processos dolorosos, comum em portadores de artrite, câncer e doenças neurodegenerativas, requer muitas vezes uma intervenção farmacológica constituída, predominantemente, por anti-inflamatórios e analgésicos de ação periférica ou central. ${ }^{3-5}$

A família Myrtaceae é uma das mais representativas, em riqueza e abundância, nas florestas tropicais e subtropicais do globo, compreendendo entre 3.100 e 4.600 espécies distribuídas em 144 gêneros, ${ }^{6}$ alguns desses com relatos de ações antimicrobiana in $v i$ tro $^{7-9} \mathrm{e}$ antioxidante, ${ }^{10}$ assim como antinociceptiva e anti-inflamatória em roedores, a exemplo de Campomanesia adamantium Camb., ${ }^{11}$ Marliera tomentosa Camb. ${ }^{12}$ Eugenia candolleana DC., ${ }^{13}$ Eugenia caryophyllata Thunb ${ }^{14}$ e Syzygium cumini L. $^{15}$

O gênero Myrcia DC. é um dos maiores da família, com mais de 300 espécies distribuídas do México até a região sul do Brasil. ${ }^{16}$ A literatura aponta alguns estudos envolvendo os efeitos antinociceptivo, em modelo animal, dos óleos essenciais obtidos das folhas de $M$. pubiflora DC. ${ }^{17}$ e $M$. ovata Cambess, ${ }^{18}$ entretanto, nenhum estudo foi realizado com $M$. rostrata DC. Assim, esse trabalho teve como objetivo analisar a composição química e avaliar o potencial antinociceptivo do óleo essencial de $M$. rostrata DC. em modelo animal.

\section{PARTE EXPERIMENTAL}

\section{Coleta}

As folhas de Myrcia rostrata DC. foram coletadas em janeiro de 2016 num remanescente de Floresta Ombrófila, localizado no Campus II da Universidade do Estado da Bahia (UNEB), município de Alagoinhas, estado da Bahia (12 10' 37' S e 38 24' 35” W). Após a expedição de coleta, o material fértil foi herborizado para a confecção da exsicata, a qual foi depositada no Herbário do Departamento de Biologia da Universidade Estadual de Feira de Santana (HUEFS) sob o número 155.923. A identificação taxonômica foi realizada por Aline do Nascimento Silva.

\section{Obtenção do óleo essencial}

O óleo essencial de $M$. rostrata (OEMr) foi obtido, em triplicata, por hidrodestilação das folhas frescas em um aparelho de Clevenger, por um período de $3 \mathrm{~h}$. Em seguida, foi seco com sulfato de sódio anidro $\left(\mathrm{Na}_{2} \mathrm{SO}_{4}\right)$, transferido para frascos de vidro âmbar e armazenado a $-22{ }^{\circ} \mathrm{C}$, até a realização da análise química e dos testes farmacológicos. $\mathrm{O}$ teor de óleo essencial foi determinado pela relação entre o volume de óleo obtido e a biomassa vegetal livre de umidade.

\section{Análise química}

A análise da composição química foi realizada pela utilização de duas técnicas: a Cromatografia em Fase Gasosa acoplada a um Detector de Ionização em Chama (CG/DIC) para a quantificação dos constituintes e a Cromatografia de Fase Gasosa acoplada a Espectrometria de Massas (CG/EM) para a avaliação qualitativa dos óleos essenciais. Na análise por CG/DIC foi usado um Cromatógrafo Varian ${ }^{\circledR}$ CP-3380, equipado com DIC e coluna capilar DB5 
(30 $\mathrm{m} \times 0,25 \mathrm{~mm}$ ), espessura do filme $0,25 \mu \mathrm{m}$, temperatura do injetor $220^{\circ} \mathrm{C}$ e do detector $240{ }^{\circ} \mathrm{C}, \mathrm{He}$ como gás de arraste e vazão de $1 \mathrm{~mL} \mathrm{~min}{ }^{-1}$, programa de temperatura do forno de: 60 a $240{ }^{\circ} \mathrm{C}$ $\left(3{ }^{\circ} \mathrm{C} \cdot \mathrm{min}^{-1}\right)$, mantendo uma isoterma de $240{ }^{\circ} \mathrm{C}$ durante $20 \mathrm{~min}$. Já na análise por CG/EM foi utilizado um Cromatógrafo Shimadzu ${ }^{\circledR}$ CG-2010 acoplado a Espectrômetro de Massas CG/MS-QP 2010 Shimadzu®, coluna capilar DB-5ms (30 m x 0,25 mm), espessura do filme $0,25 \mu \mathrm{m}$, temperatura do injetor $220^{\circ} \mathrm{C}$, gás de arraste $\mathrm{He}$ na vazão de $1 \mathrm{~mL} \mathrm{~min}^{-1}$, temperatura da interface e da fonte de ionização $240{ }^{\circ} \mathrm{C}$, energia de ionização $70 \mathrm{eV}$, corrente de ionização 0,7 $\mathrm{kV}$ e programa de temperatura do forno: 60 a $240{ }^{\circ} \mathrm{C}\left(3{ }^{\circ} \mathrm{C} \cdot \mathrm{min}^{-1}\right)$, mantendo uma isoterma de $240{ }^{\circ} \mathrm{C}$ por $20 \mathrm{~min}$. A identificação dos constituintes foi realizada através do cálculo do índice de Kovats e do índice Aritmético de cada um dos picos, obtidos pela co-injeção da amostra com uma série homóloga de n-alcanos $\left(\mathrm{C}_{8}\right.$ a $\left.\mathrm{C}_{24}\right)$, além da comparação com dados da literatura ${ }^{19}$ e com os espectros de massa da biblioteca do equipamento.

\section{Testes farmacológicos}

\section{Animais}

Em todos os experimentos, com exceção do teste de toxicidade, foram utilizados camundongos machos (Mus musculus L., 1758), jovens 3-4 meses (25-35 g), da linhagem Swiss, obtidos do Biotério Central da Universidade Estadual de Feira de Santana. Os animais foram mantidos a $22 \pm 2{ }^{\circ} \mathrm{C}$, com ciclo claro/escuro de $12 \mathrm{~h}$ e livre acesso à comida e água. Duas horas antes de cada teste, foram submetidos ao jejum. Todos os procedimentos experimentais foram previamente submetidos à Comissão de Ética no Uso de Animais da Universidade Estadual de Feira de Santana e aprovados conforme protocolo número 008/2017.

\section{Avaliação da atividade motora na barra giratória (rota rod)}

Para evitar uma interpretação equivocada dos resultados devido a uma incapacidade natural dos animais em manter o equilíbrio e a movimentação na barra giratória, $24 \mathrm{~h}$ antes da execução do teste foi realizada uma pré-seleção dos animais (sem a administração de substâncias) na qual foram considerados aptos a participar aqueles que permaneceram no equipamento (com velocidade de 7 r.p.m.) durante $180 \mathrm{~s}$, em até três tentativas. No dia seguinte, os camundongos pré-selecionados foram divididos aleatoriamente em 5 grupos $(n=8)$, sendo um grupo controle (os animais receberam o veículo, ou seja, solução salina a $0,9 \%$ com $100 \mu \mathrm{L}$ de Tween 80 a $5 \%$ por via intraperitoneal), um grupo padrão (os animais foram tratados com diazepam Hipolabor/Sanval® na dose de $4 \mathrm{mg} \mathrm{kg}^{-1}$, via i.p.) e três grupos experimentais (os animais receberam as doses de 75, $150 \mathrm{ou}$ $300 \mathrm{mg} \mathrm{kg}{ }^{-1}$ do óleo essencial de $M$. rostrata diluído no veículo por via i.p.). Após a administração das substâncias, os camundongos foram submetidos ao teste aos 30, 60 e $120 \mathrm{~min}$, para o registro do tempo de permanência na barra giratória.

\section{Teste de toxicidade oral aguda}

Para a realização do teste de toxicidade oral aguda foram utilizados camundongos fêmeas, ${ }^{20}$ jovens (25-30 g), nulíparas e não grávidas. Os animais foram marcados para permitir a identificação individual e separados em cinco grupos $(n=6)$, sendo um controle (os camundongos receberam, por via oral, solução salina a $0,9 \%$ com $100 \mu \mathrm{L}$ de Tween 80 a 5\%, num volume de $0,1 \mathrm{~mL} / 10 \mathrm{~g}$ de massa corporal) e quatro experimentais, nos quais foram administradas (v.o) as doses de 300, 500, 1000 ou $2000 \mathrm{mg} \mathrm{kg}^{-1}$ do óleo essencial de $M$. rostrata solubilizados em solução salina a $0,9 \%$ com $100 \mu \mathrm{L}$ de Tween 80 a 5\%, respeitando também o volume de $0,1 \mathrm{~mL} / 10 \mathrm{~g}$ por animal.
Os animais foram observados durante as quatro primeiras horas após a administração das substâncias, seguindo alguns dos parâmetros reunidos em um protocolo padrão, ${ }^{21}$ sendo, a posteriori, mantidos em gaiolas durante 14 dias para avaliação diária de sinais de toxicidade. A massa corporal de cada animal foi mensurada no primeiro, sétimo e décimo quarto dias, sendo a mesma comparada com os animais do grupo controle.

\section{Teste das contorções abdominais induzidas pelo ácido acético}

Esse teste se baseia no fato de que uma injeção intraperitoneal do ácido acético a $0,8 \%$ provoca uma reação nociceptiva, caracterizada por contorções abdominais seguidas de extensões dos membros posteriores. ${ }^{22}$ Para a realização do experimento, cinco grupos $(\mathrm{n}=8)$ foram formados: o controle (animais tratados com o veículo, solução salina a $0,9 \%$ com $100 \mu \mathrm{L}$ de Tween 80 a $5 \%$ via i.p.), o padrão (animais tratados com indometacina Sigma ${ }^{\circledR}$ na dose de $20 \mathrm{mg} \mathrm{kg}^{-1}$, via i.p.) e três grupos experimentais (animais tratados com o óleo essencial de $M$. rostrata nas doses de 75, 150 ou $300 \mathrm{mg} \mathrm{kg}^{-1}$, diluídos no veículo, por via i.p.). Após 30 minutos da administração das substâncias, os animais receberam uma solução de ácido acético a $0,8 \%(0,1 \mathrm{~mL} / 10 \mathrm{~g})$ também por via i.p, sendo a posteriori colocados, individualmente, em caixas de observação, para o registro do número total de contorções abdominais apresentadas durante 15 minutos, contados a partir dos 5 min iniciais após a injeção do agente álgico.

\section{Teste da formalina}

Considerado um modelo de dor associado à lesão tecidual provocada pela injeção de formalina (2,5\% de formaldeído em solução salina) na pata do camundongo, esse teste segue um padrão bifásico constituído por uma fase inicial aguda (primeira fase), e um período mais prolongado (segunda fase) de atividade comportamental aumentada. ${ }^{23}$ Para a realização do mesmo, cinco grupos $(n=8)$ foram formados: o controle (os animais receberam o veículo, solução salina a $0,9 \%$ com $100 \mu \mathrm{L}$ de Tween 80 a 5\% via i.p.), o padrão (os animais foram tratados com indometacina Sigma ${ }^{\circledR}$ na dose de $20 \mathrm{mg} \mathrm{kg}^{-1}$, via i.p.) e três grupos experimentais (os animais foram tratados com o óleo essencial de M. rostrata nas doses de 75, 150 ou $300 \mathrm{mg} \mathrm{kg}^{-1}$, diluídos no veículo, por via i.p.).

Decorridos 30 minutos da administração das substâncias, os animais receberam $20 \mu \mathrm{L}$ de formalina na região subplantar da pata posterior direita, sendo imediatamente colocados em caixas individuais de observação providas de espelhos para a contagem do tempo total gasto, em segundos, em que o animal permaneceu lambendo ou mordendo a pata injetada. Tal aferição foi realizada entre 0 e 5 min (fase 1) e entre os 15 e 30 min (fase 2) após a injeção do agente flogístico.

\section{Teste da placa quente (Hot plate)}

Esse método visa avaliar o tempo de permanência dos animais sobre uma placa metálica aquecida a uma temperatura de $54 \pm 1{ }^{\circ} \mathrm{C}$ até reagirem ao estímulo térmico com o comportamento de saltar ou lamber as patas. ${ }^{24} \mathrm{~A}$ fim de evitar uma interpretação equivocada dos resultados, um dia antes do experimento, os camundongos foram pré-selecionados (sem a administração de substâncias), sendo considerados aptos, aqueles que permaneceram na placa aquecida por mais de 10 e menos de $30 \mathrm{~s}$.

No dia do teste, os animais foram divididos aleatoriamente em cinco grupos ( $\mathrm{n}=8)$, o controle (os camundongos receberam o veículo, solução salina a $0,9 \%$ com $100 \mu \mathrm{L}$ de Tween 80 a 5\%, via i.p.), o padrão (foi administrada morfina Cristália ${ }^{\circledR}$ na dose de $10 \mathrm{mg} \mathrm{kg}^{-1}$, via i.p.) e três grupos experimentais (os animais foram tratados com as doses de 75, 150 ou $300 \mathrm{mg} \mathrm{kg}^{-1}$ do óleo essencial de $M$. rostrata). 
Em seguida, os camundongos foram colocados, individualmente, na superfície da placa aos 30, 60, 90 e 120 minutos após a administração das substâncias. O intervalo decorrido entre a colocação do animal na placa e a ocorrência de lambida das patas ou comportamento de pular foi registrado em segundos. Um tempo de corte de $30 \mathrm{~s}$ foi utilizado para indicar analgesia completa e evitar lesão tecidual.

\section{Análise estatítica}

Os dados foram submetidos à análise de variância de uma via, seguida pelo pós teste de Dunnett. Os resultados foram representados como média \pm erro padrão da média e considerados significativos quando $\mathrm{p}<0,05$.

\section{RESULTADOS E DISCUSSÃO}

\section{Análise do óleo essencial}

O óleo essencial de $M$. rostrata apresentou um rendimento de $0,42 \%(\mathrm{~m} / \mathrm{v})$, mais do que o dobro do obtido $(0,2 \%)$ a partir das folhas frescas dessa mesma espécie coletadas em populações nativas do Rio Grande do Sul. ${ }^{25}$ A análise da composição química revelou que do total dos constituintes químicos identificados $(87,62 \%)$; $4,76 \%$ corresponderam a monoterpenos e $95,24 \%$ a sesquiterpenos. Dentre estes últimos, destacaram-se o dauceno (4,7\%), germacreno D $(5,68 \%), E$-cariofileno $(6,45 \%)$ e o germacreno B $(7,28 \%)$, sendo o carotol $(17,68 \%)$, o composto majoritário (Tabela 1). Esta composição química difere da descrita para o óleo essencial da espécie nativa do Rio Grande do Sul, com 93,3\% de sesquiterpenos, tendo $\alpha$-cadinol (4,6\%), $\delta$-cadineno $(5,7 \%)$ e óxido de cariofileno $(13,1 \%)$ como constituintes majoritários, ${ }^{25}$ assim a população oriunda da Bahia pode se tratar de um novo quimiotipo de $M$. rostrata.

A predominância de sesquiterpenos nos óleos essenciais de outras espécies de Myrcia também foi anteriormente destacada. ${ }^{17,26-28}$ Do óleo essencial de $M$. cuprea coletada no Pará, por exemplo, obteve-se como constituintes majoritários $\beta$-cariofileno $(38,1 \%)$, germacreno $\mathrm{D}(21,8 \%)$ e germacreno B $(19,5 \%) .{ }^{29}$ Num estudo comparativo com os óleos essenciais de diferentes espécies de Myrcia foi detectada a predominância de sesquiterpenos cíclicos, principalmente os derivados das vias de ciclização do cadinano, germacrano e cariofilano. ${ }^{25}$ Por outro lado, o óleo obtido das folhas de M. ovata Cambess apresentou os monoterpenos oxigenados como a mais abundante classe de compostos, correspondendo a $91,78 \%$ do total de identificados. ${ }^{18}$

\section{Avaliação da coordenação motora na barra giratória (rota-rod)}

Os camundongos tratados com as doses de 75, 150, ou $300 \mathrm{mg} \mathrm{kg}^{-1}$ do OEMr não exibiram diferença estatística significativa quanto ao tempo de permanência na barra giratória, ao serem comparados com o grupo que recebeu apenas o veículo, nos três tempos avaliados (Figura 1).

Levando em consideração que esse método avalia a integridade do sistema motor, permitindo a detecção de enfraquecimento neurológico, abrangendo incoordenação motora e ralaxamento muscular, consequências comuns de neurotoxidade, ${ }^{30,31}$ pode-se afirmar que os constituintes químicos presentes no óleo não interferiram na capacidade motora dos animais nem provocaram ação miorrelaxante.

Como esperado, os murinos que receberam diazepam na dose de $4 \mathrm{mg} \mathrm{kg}^{-1}$ (i.p), droga conhecida por sua atividade depressora do $\mathrm{SNC}$, tiveram seu tempo médio de permanência na barra giratória reduzido aos 60 e $120 \mathrm{~min}(46,75 \pm 5,81 \mathrm{~s}$ e $71,13 \pm 14,04 \mathrm{~s}$, respectivamente) e de forma mais acentuada após $30 \mathrm{~min}$ da administração (26,63 $\pm 13,46 \mathrm{~s})$ quando comparado aos valores médios do grupo controle $\left(\mathrm{T}_{30}=165,5 ; \mathrm{T}_{60}=180\right.$ e $\left.\mathrm{T}_{120}=165,4 \mathrm{~s}\right)$.
Tabela 1. Análise da composição química do óleo essencial obtido das folhas frescas de Myrcia rostrata DC. (MYRTACEAE)

\begin{tabular}{|c|c|c|c|}
\hline Composto & $\mathrm{IK}_{\mathrm{lit}}$ & $\mathrm{IK}_{\text {calc }}$ & $\begin{array}{c}\text { Percentual relativo } \\
(\%)\end{array}$ \\
\hline$\alpha$-pineno & 939 & 943 & $1,97 \pm 0,31$ \\
\hline$\beta$-pineno & 979 & 989 & $1,1 \pm 0,21$ \\
\hline$\beta$-mirceno & 990 & 994 & $0,4 \pm 0,09$ \\
\hline limoneno & 1029 & 1041 & $0,27 \pm 0,04$ \\
\hline$\alpha$-terpineol & 1188 & 1212 & $0,41 \pm 0,05$ \\
\hline$\alpha$-cubebeno & 1348 & 1357 & $0,15 \pm 0,13$ \\
\hline isoledeno & 1376 & 1383 & $0,27 \pm 0,01$ \\
\hline$\alpha$-copaeno & 1376 & 1387 & $3,02 \pm 0,14$ \\
\hline dauceno & 1381 & 1389 & $4,7 \pm 0,54$ \\
\hline$\beta$-elemeno & 1389 & 1399 & $0,26 \pm 0,01$ \\
\hline$\alpha$-cis-bergamoteno & 1412 & 1423 & $0,30 \pm 0,005$ \\
\hline$E$-cariofileno & 1419 & 1436 & $6,45 \pm 1,31$ \\
\hline$\alpha$-trans-bergamoteno & 1434 & 1443 & $4,75 \pm 0,31$ \\
\hline aromadendreno & 1441 & 1451 & $0,42 \pm 0,3$ \\
\hline epi- $\alpha$-santaleno & 1447 & 1455 & $0,79 \pm 0,02$ \\
\hline$\beta$-farneseno & 1456 & 1459 & $3,72 \pm 0,26$ \\
\hline$\alpha$-humuleno & 1454 & 1472 & $0,71 \pm 0,06$ \\
\hline dauca-5,8-dieno & 1472 & 1476 & $0,97 \pm 0,02$ \\
\hline amorfa-4,7(11)-dieno & 1481 & 1487 & $4,98 \pm 0,49$ \\
\hline germacreno D & 1485 & 1496 & $5,68 \pm 0,84$ \\
\hline viridifloreno & 1496 & 1506 & $3,35 \pm 0,09$ \\
\hline biciclogermacreno & 1500 & 1512 & $4,73 \pm 0,22$ \\
\hline$\beta$-bisaboleno & 1505 & 1520 & $4,46 \pm 0,42$ \\
\hline$\gamma$-cadineno & 1513 & 1533 & $3,33 \pm 0,31$ \\
\hline$\beta$-sesquifelandreno & 1522 & 1537 & $3,34 \pm 0,22$ \\
\hline germacreno B & 1561 & 1580 & $7,28 \pm 2,65$ \\
\hline espatulenol & 1578 & 1598 & $0,36 \pm 0,1$ \\
\hline óxido de cariofileno & 1583 & 1600 & $\mathrm{t}$ \\
\hline viridiflorol & 1592 & 1608 & $1,67 \pm 0,03$ \\
\hline carotol & 1594 & 1627 & $17,68 \pm 1,45$ \\
\hline \multicolumn{3}{|c|}{ Total de compostos identificados } & 87,36 \\
\hline
\end{tabular}

Resultados expressos como média \pm desvio padrão, $\mathrm{t}=$ traços .

\section{Toxicidade oral aguda}

Os resultados indicaram que mesmo para os animais que foram tratados com a dose de $2000 \mathrm{mg} \mathrm{kg}^{-1}$ do OEMr não houve sinais de toxidade evidente nem mortes durante os 14 dias de observação. A massa corporal dos animais no início, no sétimo dia e no final do teste, não apresentou diferença estatística significativa na análise de variância ao nível de 5\% de significância, quando comparada com o grupo controle.

\section{Teste das contorções abdominais induzidas pelo ácido acético}

Os dados exibidos na Figura 2 demonstram que o OEMr apresentou efeito de antinocicepção bastante significativo $(\mathrm{p}<0,0001)$, com números de contorções abdominais iguais a 6,$87 ; 1,75 \mathrm{e}$ 1,62 nos grupos tratados com as doses de 75, 150 ou $300 \mathrm{mg} \mathrm{kg}^{-1}$, 
$30 \mathrm{~min}$

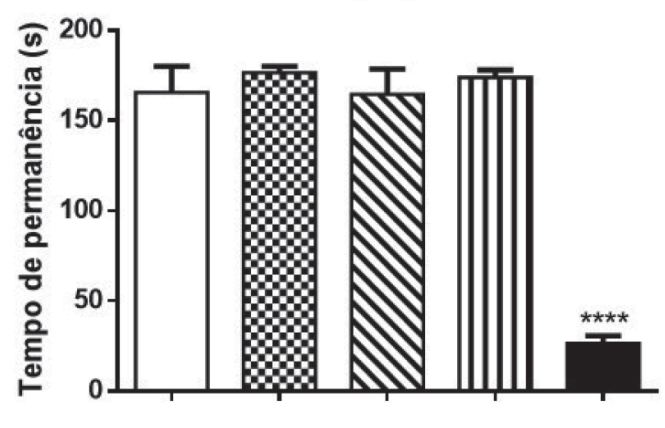

Tratamentos
$60 \mathrm{~min}$

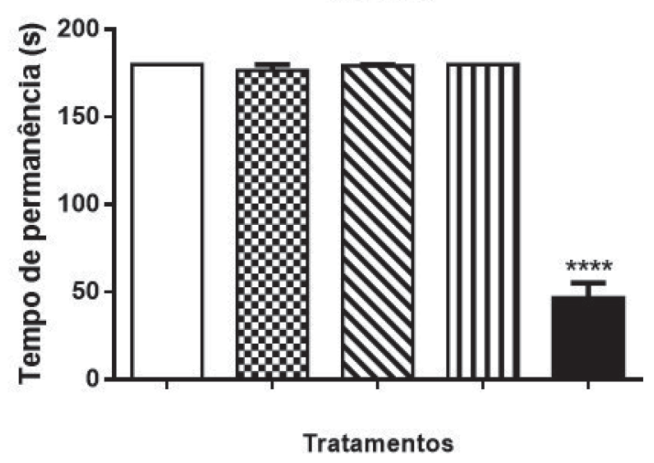

$120 \mathrm{~min}$

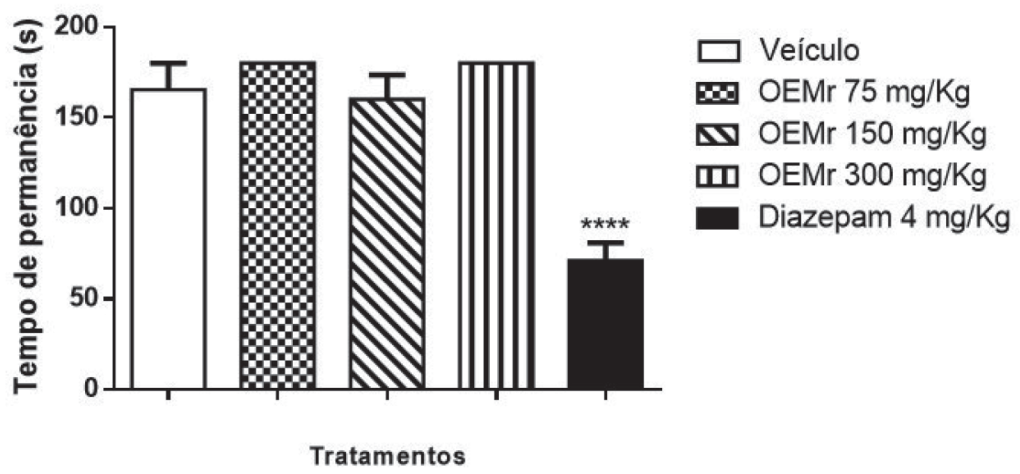

Figura 1. Efeito do óleo essencial de Myrcia rostrata na coordenação motora dos camundongos aos 30 , 60 e 120 min após a administração das substâncias. Resultados expressos como média \pm e.p.m. **** p<0,0001 indica diferença significativa quando comparado ao grupo controle. (ANOVA seguido de Dunnett, $n=8$ por grupo)

respectivamente, valores bastante reduzidos quando comparados à média do grupo controle $(27,88 \pm 2,75)$.

A indometacina, na dose de $20 \mathrm{mg} \mathrm{kg}^{-1}$ (i.p.), ocasionou uma redução também significativa $(\mathrm{p}<0,0001)$ e semelhante à maior dose do óleo essencial, com valor médio de 1,60 $\pm 2,75$ contorções. A análise de variância também revelou que não houve diferença estatística entre os tratamentos entre si, tampouco entre os tratamentos e a indometacina, num intervalo de confiança de $95 \%$.

A literatura descreve que a dor inflamatória está intimamente relacionada à ativação de nociceptores polimodais sensíveis tanto à prótons (a exemplo dos canais iônicos sensíveis à ácido, conhecidos pela sigla em inglês ASICs), quanto à bradicinina, prostaglandina e citocinas. ${ }^{32,33}$ Sabe-se que tal estimulação segue pela via aferente (mediada por fibras tipo C) até os impulsos atingirem o SNC. ${ }^{18}$

Alguns autores consideram a resposta nociceptiva mediada pelo ácido acético como inespecífica e de baixa seletividade, ademais tanto drogas de ação central quanto periférica podem atuar inibindo o número de contorções, assim como anti-histamínicos e anti-colinérgicos, ${ }^{34,35}$ o que de certa forma dificulta eleger determinada substância como analgésica sem que testes com modelos mais específicos, a exemplo do teste da formalina, sejam realizados.

Além disso, seria pretensioso atribuir a proeminente ação do OEMr na redução do número de contorções abdominais à inibição de substâncias endógenas que atuam no estímulo de fibras nervosas associadas à nocicepção, ao bloqueio dos nociceptores ASICs ou à via dos prostanoides pela inibição da COX (mecanismo pelo qual a indometacina atua), sem que testes mais específicos fossem realizados.

\section{Teste da formalina}

O OEMr nas doses de 150 e $300 \mathrm{mg} \mathrm{kg}^{-1}$ reduziu de forma significativa o tempo de lambidas e mordidas na pata dos animais,

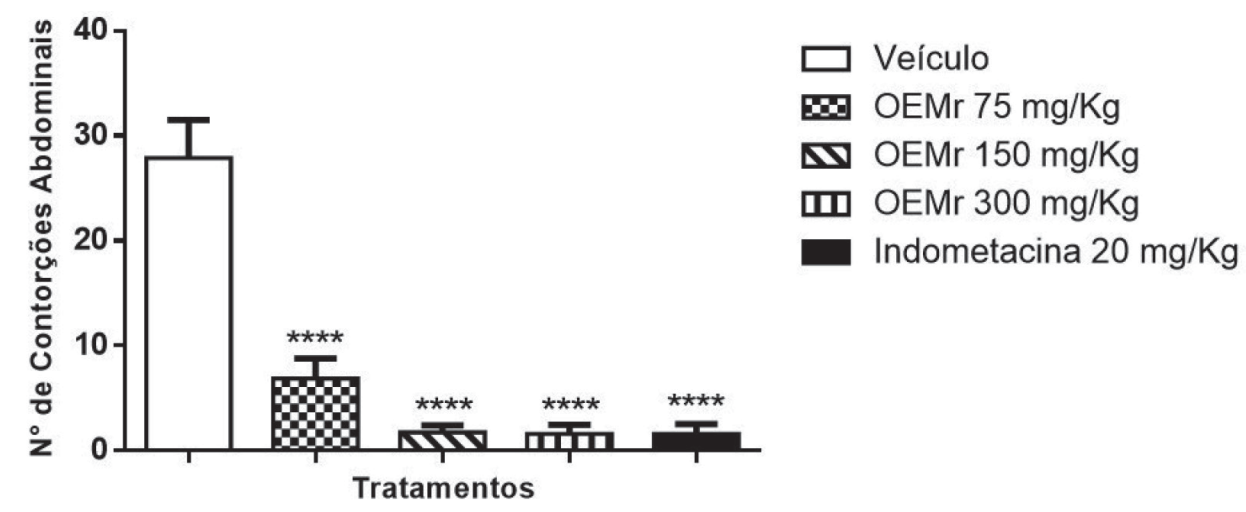

Figura 2. Efeito do óleo essencial de Myrcia rostrata no teste das contorções abdominais induzidas pelo ácido acético. Resultados expressos como média \pm e.p.m. $* * * * p<0,0001$ e comparados com o grupo controle. (ANOVA seguido de Dunnett) 
na fase 1 (neurogênica), com valores de 26,88 e 21,5 $\pm 8,3$ s, respectivamente, quando comparado ao grupo controle $(55,88 \mathrm{~s})$. Na fase 2 (inflamatória), as três doses foram capazes de inibir o tempo total gasto em lambidas com valores de 18,$13 ; 9,75$ e 4,75 $\pm 12,75$ s, respectivamente (Figura 3), quando comparadas aos animais do grupo controle $(64,75 \mathrm{~s})$. Vale ressaltar que não houve diferença estatística significativa entre os grupos tratados com o óleo e o grupo tratado com a indometacina na dose de $20 \mathrm{mg} \mathrm{kg}^{-1}$ (tempo de lambida de 20,13 s).

A inibição no tempo de lambida, na fase neurogênica, evidenciada nos grupos que receberam as doses de 150 e $300 \mathrm{mg} \mathrm{kg}^{-1}$ do OEMr corrobora os resultados obtidos no teste das contorções abdominais induzidas pelo ácido acético. É provável que a ação do óleo esteja relacionada à inibição química direta de fibras aferentes nociceptivas, que podem ser suprimidas por analgésicos opioides como a morfina, ${ }^{36}$ isso pode ser comprovado mediante a realização de experimentos com modelos que utilizem antagonistas de receptores $\mu$-opioides como a naloxona. A segunda fase do teste da formalina está relacionada à liberação de mediadores da inflamação, e dessa forma, a maioria dos anti-inflamatórios não esteroides (AINEs), a exemplo do ácido acetilsalicílico, da indometacina e do naproxeno atua minimizando o tempo de lambida e/ou mordidas. ${ }^{32,37}$ Vale ressaltar que na fase inflamatória, o óleo essencial de $M$. rostrata exibiu resultados significativos nas três doses, com destaque para a de $300 \mathrm{mg} \mathrm{kg}^{-1}$, que reduziu em 13,63 vezes o tempo de lambida da pata quando comparado ao grupo controle.

Em síntese, o teste da formalina reproduz um modelo mais válido de dor clínica quando comparado aos que levam em conta estímulos térmicos por exemplo, ${ }^{38}$ especialmente, por seguir um padrão bifásico. ${ }^{23}$ Partindo dessa premissa, sua utilização pode auxiliar na detecção de drogas com ações tanto analgésicas, sejam estas de ação periférica ou central, quanto anti-inflamatórias.

\section{Placa quente (Hot plate)}

Os resultados obtidos demonstraram que o OEMr, após 30 min da sua administração (Figura 4), promoveu um aumento da latência em resposta ao estímulo térmico, quando comparado ao grupo controle $\left(\mathrm{T}_{30}: 11 \mathrm{~s}\right)$ com destaque para as doses de 150 e $300 \mathrm{mg} \mathrm{kg}^{-1}\left(\mathrm{~T}_{30}\right.$ : $75 \mathrm{mg} \mathrm{kg}^{-1} 18,75 ; 150 \mathrm{mg} \mathrm{kg}^{-1}, 23,38$ e $300 \mathrm{mg} \mathrm{kg}^{-1}, 25,5 \pm 2,8 \mathrm{~s}$ ).

Aos $60 \mathrm{~min}$, o mesmo efeito foi mantido em relação ao controle $\left(\mathrm{T}_{60}: 14,25 \mathrm{~s}\right)$, com tempos de latência de $\left(\mathrm{T}_{60}: 75 \mathrm{mg} \mathrm{kg}^{-1}, 18,38\right.$; $150 \mathrm{mg} \mathrm{kg}^{-1}, 22,75$ e $300 \mathrm{mg} \mathrm{kg}^{-1}, 26,75 \pm 2,19$ s). Aos 90 min, não houve diferença estatística significativa entre os tratamentos e a morfina e aos 120 min o aumento da latência persistiu em relação ao controle $\left(\mathrm{T}_{120}: 14,1 \mathrm{~s}\right)$ para a dose de $300 \mathrm{mg} \mathrm{kg}^{-1}\left(\mathrm{~T}_{120}: 26,5 \pm 3,5 \mathrm{~s}\right)$, sendo inclusive superior $(\mathrm{p}<0,01)$ ao da morfina na dose de $10 \mathrm{mg} \mathrm{kg}^{-1}$ $\left(\mathrm{T}_{120}: 22,75 \pm 3,5 \mathrm{~s}\right)$.

Os animais que receberam a dose de $300 \mathrm{mg} \mathrm{kg}^{-1}$ tiveram um aumento na latência aos $30 \mathrm{~min}$ duas vezes maior do que os do grupo controle. Aos $120 \mathrm{~min}$, não se observou diferença estatística significativa entre os animais tratados com a morfina e os que receberam apenas o veículo, entretanto, o grupo tratado com a maior dose do óleo essencial apresentou uma resposta 1,87 vezes maior do que o controle. Aos $240 \mathrm{~min}$, nenhum dos tratamentos apresentou diferença estatística significativa em relação ao grupo controle, o que provavelmente está relacionado ao clearance das substâncias, inclusive da morfina.

Outros óleos de Myrcia foram avaliados nesse modelo, a exemplo do óleo essencial de $M$. publiflora, constituído majoritariamente por óxido de cariofileno (22,2\%), mustacona (11,3\%), 1,8-cineol $(5,4 \%)$ e tricicleno $(5,3 \%)$, o qual foi ineficaz na inibição do tempo de reação dos animais ao estímulo térmico nas doses de 25,50 e $100 \mathrm{mg} \mathrm{kg}^{-1} \cdot{ }^{17}$

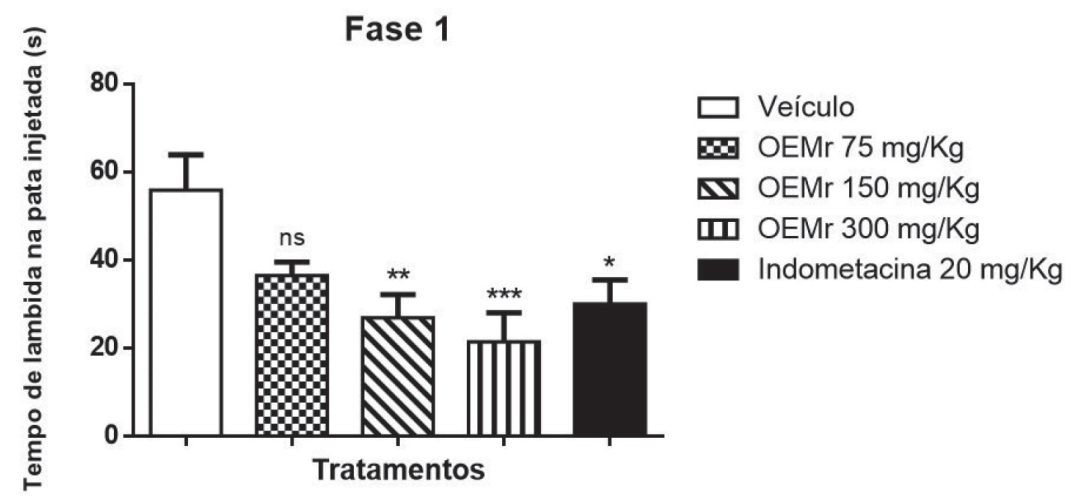

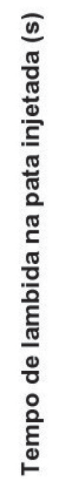

Fase 2

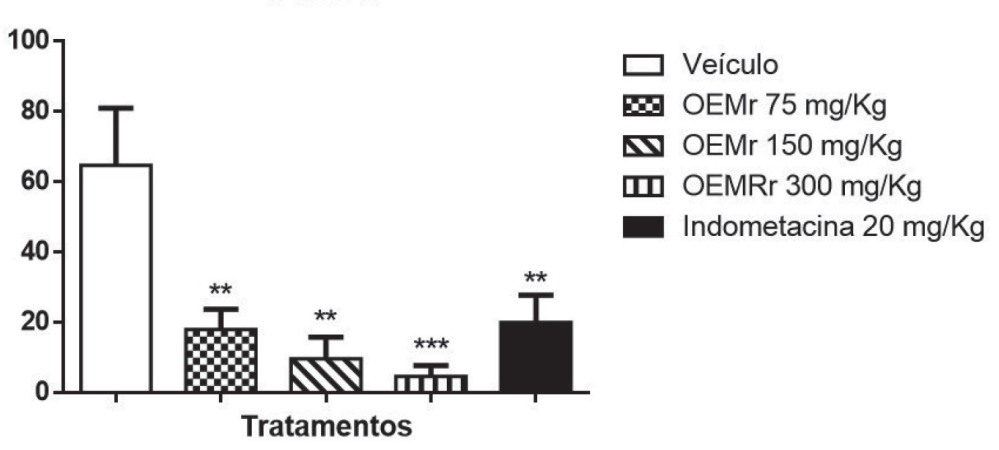

Figura 3. Efeito do óleo essencial de Myrcia rostrata no teste da formalina. Resultados expressos como média \pm e.p.m. ns = não significativo; ** p<0,01; *** p<0,001. (ANOVA seguido de Dunnett) 
$30 \mathrm{~min}$

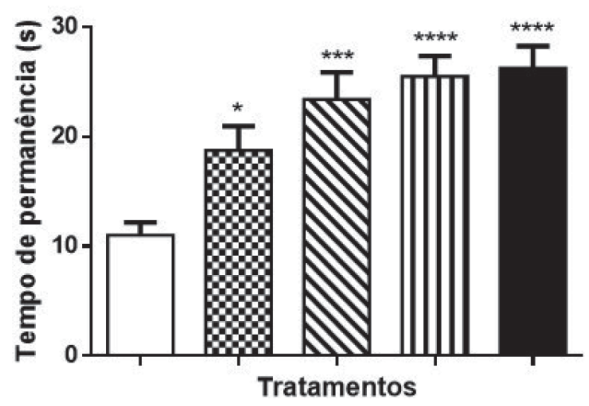

$90 \mathrm{~min}$

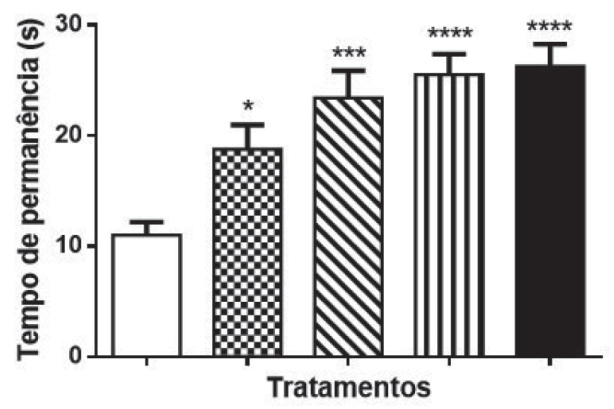

$60 \mathrm{~min}$

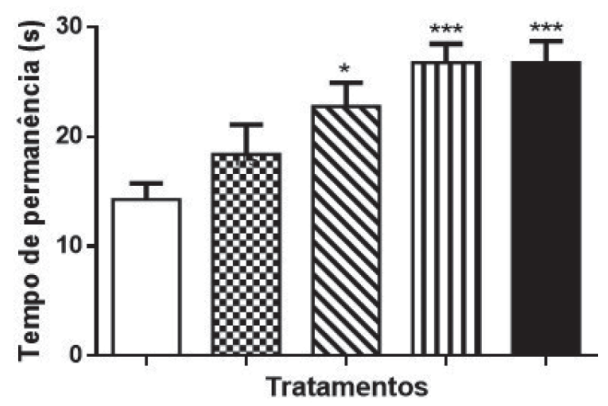

$120 \min$

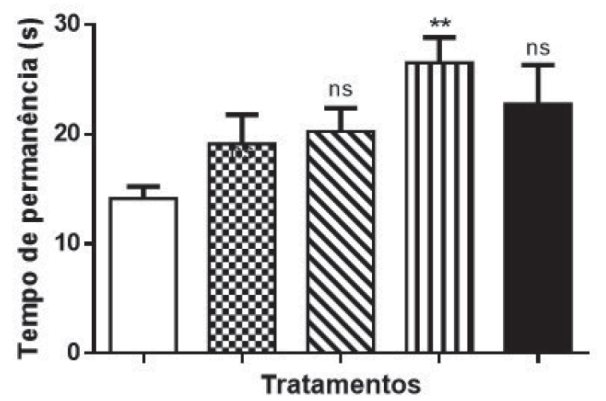

$240 \min$

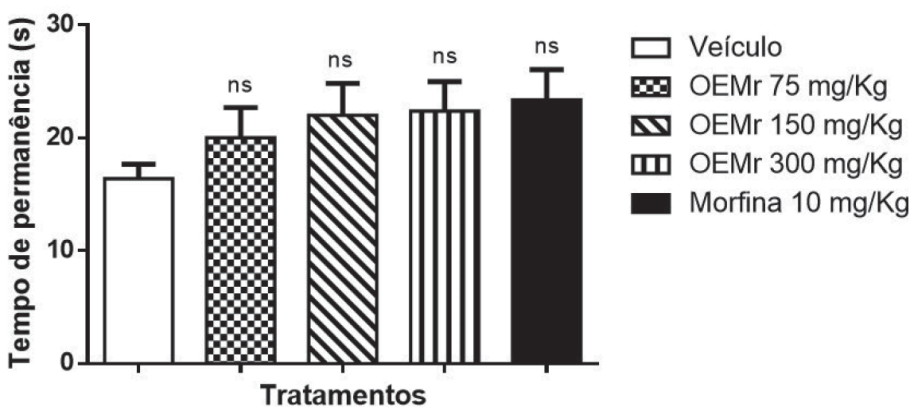

Figura 4. Efeito do óleo essencial de Myrcia rostrata no teste da placa quente avaliado aos 30, 60, 90, 120 e 240 min após a administração das substâncias. Resultados expressos como média \pm e.p.m e comparados com o grupo controle. ns = não significativo; * p<0,05; ** p<0,01; *** p<0,001; **** p<0,0001. (ANOVA seguido de Dunnett)

Sabe-se que as fibras C e A $\delta$ tipo II são estimuladas após a ativação de receptores vaniloides, especialmente os do tipo TRPV-1 quando submetidos a temperaturas entre 45 e $53^{\circ} \mathrm{C} .3^{39,40}$ Levando em consideração que os animais são expostos à superfície do equipamento aquecida aos $54 \pm 1{ }^{\circ} \mathrm{C}$, poder-se-ia conjecturar que a ação do OEMr, principalmente na dose de $300 \mathrm{mg} \mathrm{kg}^{-1}$, ao aumentar o tempo de permanência dos animais sobre a placa ocorre pelo bloqueio dos receptores térmicos ou pela inibição do estímulo nervoso aferente.

Diante de resultados tão expressivos, é notória a necessidade de posterior investigação do mecanismo de ação (se central ou periférico) do referido óleo essencial, seja pela utilização de antagonistas de receptores $\mu$-opioides como a naloxona, ou por intermédio de agonistas dos receptores vaniloides como o cinamaldeído ou o peróxido de hidrogênio $\left(\mathrm{H}_{2} \mathrm{O}_{2}\right)$, tendo em vista que estudos pré-clínicos com roedores demonstraram que antagonistas de receptores catiônicos não seletivos de potencial transitório agem inibindo tanto a nocicepção como o processo inflamatório. ${ }^{41}$

\section{CONCLUSÃO}

A análise química do óleo essencial de Myrcia rostrata DC. revelou uma predominância de sesquiterpenos, com carotol, germacreno $\mathrm{D}$ e $\mathrm{B},(E)$-cariofileno e dauceno como constituintes majoritários, indicando se tratar de um novo quimiotipo. O OEMr não demonstrou sinais de toxicidade de acordo com o preconizado pela OECD, nem ação depressora no SNC, uma vez que não houve redução na coordenação motora dos animais frente aos critérios do teste rota rod. Os resultados obtidos nos testes farmacológicos evidenciaram a significativa propriedade antinociceptiva dos constituintes químicos presentes no óleo com a necessidade de realização de experimentos que avaliem seu mecanismo de ação.

\section{REFERÊNCIAS}

1. Rang, H. P.; Dale, M. M.; Ritter, J. M.; Flower, R. J.; Henderson, G.; Rang \& Dale Farmacologia, 8 ${ }^{\mathrm{a}}$ ed., Elsevier: Rio de Janeiro, 2016. 
2. Fornasari, D.; Coaccioli, S.; Reumatismo 2014, 66, 14.

3. Rao, Y. K.; Fang, S. H.; Tzeng, Y. M.; Phytother. Res. 2008, 22, 957.

4. Dinarello, C. A.; Cell 2010, 140, 935.

5. Ekor, M.; Front. Pharmacol. 2014, 14, 1.

6. Judd, W. S.; Campbell, C. S.; Kellog, E. A.; Stevens, P. F.; Donoghue, M. J.; Plant systematics: a phylogenetic approach, $3^{\text {rd }}$ ed., Sinauer Associates: Sunderland, 1999.

7. Pavan, F. R.; Leite, C. Q. F.; Coelho, R. G.; Coutinho, I. D.; Honda, N. K.; Cardoso, C. A. L.; Vilegas, W.; Leite, S. R. de A.; Sato, D. N.; Quim. Nova 2009, 32, 1222.

8. Silva, C. J.; Barbosa, L. C. A.; Demuner, A. J.; Montanari, R. M.; Pinheiro, A, L.; Dias, I.; Andrade, N. J.; Quim. Nova 2010, 33, 104.

9. Silva, A. do N.; Uetanabaro, A. P. T; Lucchese, A. M.; Nat. Prod. Commun. 2013, 8, 269.

10. de Carvalho Júnior, A. R.; Gomes, G. A.; Ferreira, R. O.; de Carvalho, M. G.; Quim. Nova 2014, 37, 477.

11. Vendruscolo, G. S.; Rates, S. M. K.; Mentz, L. A.; Rev. Bras. Farmacogn. 2005, 15, 361.

12. Messias, K. L. S.; Campos-Buzzi, F. de; Fischer, L. G. O.; Malheiros, A.; Monache, F. D.; Filho, V. C.; Quim. Nova 2008, 31, 1747.

13. Guimarães, A. G.; Melo, M. S.; Bonfim, R. R.; Passos, L. O.; Machado, S. M. F.; Ribeiro, A. de S.; Sobral, M.; Thomazzi, S. M.; QuintansJúnior, L. J.; Rev. Bras. Farmacogn. 2009, 19, 883.

14. Daniel, A. N.; Sartoretto, S. M.; Schmidt, G.; Caparroz-Assef, S. M.; Bersani-Amado, C. A.; Cuman, R. K. N.; Rev. Bras. Farmacogn. 2009, $19,212$.

15. Lima, L. A.; Siani, A. C.; Brito, F. A.; Sampaio, A. L. F.; Henriques, M das G. M O.; Riehl, C. A. da S.; Quim. Nova 2007, 30, 860.

16. Sobral, M.; A Família Myrtaceae no Rio Grande do Sul, Unisinos: São Leopoldo, 2003.

17. Andrade, G. S.; Santana, M. T.; Siqueira, R. S.; Passos, L. O.; Machado, S. M. F.; Ribeiro, A. de S.; Sobral, M.; Almeida, J. R. G. S; QuintansJúnior, L. J.; Rev. Bras. Farmacogn. 2012, 22, 181.

18. dos Santos, G. C. M.; Gomes, G. A.; Gonçalves, G. M.; de Sousa, L. M; Santiago, G. M. P.; de Carvalho, M. G.; Marinho, B. G.; Planta Med. 2014, $80,1588$.

19. Adams, R. P.; Identification of Essential Oil Components by Gas Chromatography/Mass Spectrometry, $4^{\text {th }}$ ed., Allured Publishing Corporation: Carol Stream, 2007.

20. Organization for Economic Co-Operation and Development, Guidelines for Testing of Chemicals, OECD 423, Acute oral Toxicity, OECD: Paris, 2001.
21. Almeida, R. N.; Psicofarmacologia: fundamentos práticos, $1^{\text {a }}$ ed., Guanabara Koogan: Rio de Janeiro, 2006.

22. Koster, R.; Anderson, M.; De Beer, E. J.; Fed. Proc. 1959, 18, 412.

23. Dubuisson, D.; Dennis, S. G.; Pain 1977, 4, 161.

24. Woolfe, G.; MacDonald, A. D.; J. Pharmacol. Exp. Ther. 1944, 80, 300.

25. Limberger, R. P.; Sobral, M.; Henriques, M. E. G.; Menut, A. T.; JeanMarie, C. B.; Quim. Nova. 2004, 27, 916.

26. Cerqueira, M. D. de; Marques, E. de J.; Martins, D.; Roque, N. F.; Cruz, F. G.; Silva, M. L.; Quim. Nova 2009, 32, 1544.

27. Nakamura, M. J.; Biochem. Syst. Ecol. 2010, 38, 1170.

28. Sá, F. A. S.; Borges, L. L; Paula, J. A. M.; Sampaio, B. L.; Ferri, P. H.; Paula, J. R.; Rev. Bras. Farmacogn. 2012, 22, 1233.

29. Zoghbi, M. das G. B.; Andrade, E. H. A.; Silva, M. H. L.; Carreira, L. M. M.; Maia, J. G. S.; Flavour Fragrance J. 2003, 18, 421.

30. Mattei R.; Franca, C. I. F. Em Psicofarmacologia: Fundamentos Práticos; de Almeida, R. N., eds.; Guanabara Koogan: Rio de Janeiro, 2006, cap. 12.

31. Pultrini, A. M.; Galindo, L. A.; Costa, M.; Life Sci. 2006, 78, 1720.

32. Tjolsen, A.; Berge, O. G.; Hunskaar, S.; Rosland, J. H.; Hole, K.; Pain 1992, 51, 5 .

33. Ikeda, Y.; Ueno, A.; Naraba, H.; Oh-ishi, S.; Life Sci. 2001, 89, 2911.

34. Quintão, N. L. M.; Campos, M. M.; Calixto, J. B. Em Protocolos em Psicofarmacologia Comportamental; Carlini, E. A., Mendes, F. R., eds.; Fap-Unifesp: São Paulo, 2011, cap. 7.

35. Hayes, A. G.; Sheehan, M. J.; Tyers, T. B.; Br. J. Pharmacol. 1987, 91, 823.

36. do Amaral, J. F.; Silva, M. I. G.; de Aquino Neto, M. R.; Teixeira Neto, P. F.; Moura, B. A.; de Melo, C. T. V.; de Araújo, F. L. O.; de Sousa, D. P.; de Vasconcelos, P. F.; de Vasconcelos, S. M. M.; de Sousa, F. C. F.; Biol. Pharm. Bull. 2007, 30, 1217.

37. Dai, Y.; Ye, W. C.; Wang, Z. T.; Matsuda, H.; Kubo, M.; But, P. P. H.; J. Ethnopharmacol. 2002, 81, 245.

38. Hunskaar, S.; Hole, K.; Pain 1987, 30, 103.

39. Julius, D.; Basbaum, A. L.; Nature 2001, 413, 203.

40. Almeida, F. R. C.; Oliveira, F. S. Em Psicofarmacologia: fundamentos práticos; Almeida, R. N., eds.; Guanabara Koogan: Rio de Janeiro, 2006, cap. 17.

41. Andrade, E. L.; Meotti, F. C.; Calixto, J. B.; Pharmacol. Ther. 2012, 133, 189. 